\title{
The Enlightenment of Uber's User Experience-Centered Crossover Marketing
}

\author{
Guicheng $\mathrm{Hu}$
}

\author{
E-commerce School of Wuhan Technology and Business University, Wuhan, China \\ 14987620@qq.com
}

\section{Keywords: Crossover marketing; User experience; Uber; Brand}

\begin{abstract}
With the deepening of globalization and pluralism, the business limits and scopes of some industries and enterprises are no longer very clear and explicit; that is, the era of competition based solely on single enterprise, single brand and single product is over. Nowadays, marketing needs innovation, so crossover marketing came into being and has drawn a lot of attention in academia and business circle. This paper, based on the perspective of the user experience, introduces the general situation of crossover marketing at home and abroad, and through the investigation and analysis of the crossover marketing of the users, it also points out that the implementation of crossover marketing must focus on users and choose high-quality brands for cooperation, so as to break the traditional mode and adapt to local conditions. All in all, this paper is believed to be able to provide a reference to domestic enterprises.

With the continuous development of market economy, the global economic integration continues to be deepened. Each enterprise is involving in the marketing competition in order to occupy more market share, but with the development of the times, the business limits and scopes of some industries and enterprises are no longer very clear and explicit. Crossover marketing came into being and has drawn a lot of attention in academia and business circle.
\end{abstract}

\section{Implications of Crossover Marketing}

In 1966, American scholar Adler put forward the concept of "symbiotic marketing", and he believes that different organizations can together choose to build a resources-sharing system to enhance their own and respective marketing potential via such relationship, so as to achieve a symbiotic relationship as perfect as that in the biological world. Crossover marketing is about gathering the resources advantages of all parties in the cooperation in all aspects to obtain a marketing effect like "1+1>2". Through the common analysis and relationship exploration of different industries, enterprises, products and audiences, according to their own brand characteristics and competitive advantages, the most important parts are sought to form a mutual-benefit type of marketing by targeted focusing on the prioritized demand points of the audience, so as to strengthen the preferences of the target group on the products and achieve a mutual-benefit and win-win outcome between the two sides of cooperation.

The development of the Internet provides a prerequisite for crossover marketing. Crossover marketing has the following advantages: 1 . Resource sharing. Crossover is conducive to getting rid of inherent thinking pattern and creating a new type of marketing thinking, using different means to find different partners, so as to achieve the sharing of related resources. 2. Promoting the social effect of the brand. Crossover marketing enhances the social effect of the brand, through the way of cross penetration; it effectively attracts potential customers, gains the unified preference of the target group, and then enlarges the competition strength of the brand. 3. Reducing marketing costs and improving marketing efficiency. Crossover marketing is a low-cost, good-efficiency and high-coverage marketing tool with a wide range of audiences. The cost of marketing under crossover pattern is much lower than it used to be, and it can also achieve a marketing effect far better than that of any traditional means. An enterprise just needs to cooperate by standing in the position of integrating its own resources, but needs no additional costs, so it seems that the benefits will obviously increase. 


\section{The Role of Crossover Marketing in User Experience}

The user experience lies in their subjective psychological feeling. The approbation degree of users on the product is completely subject to their subjective consciousness. Different users may have different experiences, so the enterprise must look for the common ground of different users for the excavation of user needs, so as to achieve the specialized marketing. As a new marketing model, the function of crossover marketing on user experience can be divided into the following two aspects:

First, the number of new-type users in the development of crossover marketing is growing. The demands of new-type users on whether tangible or intangible products are no longer limited to functionality, but rather hope that the products will bring them more experience in life and work.

Second, crossover marketing has focused more on the segmentation of user experience. Market competition exists because that in the open market environment, whether products or marketing methods are consistent to a certain extent. These factors require companies to focus more on users than on competitors. Because the focusing subjects have been shifted from the products to the users, the user experience must be subdivided, and the impact factors are no longer simple factors such as age or education, but are partly shifted to individual differences.

To sum up, the user experience enables crossover marketing to achieve a leap in the marketing dimension, to make a deeper excavation of the demand, and to help the audience reach the realization of the perfect transformation from simple physical pleasure to a variety of inner experience, so that the brand effect of the enterprise can be quickly improved and the image of the enterprise can be more acceptable to the audiences. Both sides of the partnership can also receive a certain amount of income. This form eliminates the drawbacks of the traditional model of fighting alone, and promotes the integration between brands and the target groups.

\section{Overview on the Crossover Marketing of Uber}

Uber, founded in the United States in 2010, is successfully developed and growing with a variety of marketing tools. In the 68th session of the Cannes Film Festival, Uber carried out activities of helicopter services for visitors to draw public attention; in Japan cherry blossom season, for the pollen-allergy crowd, it carried out activities of "Child pollen allergy is no longer a problem" and used drones to send the mass masks, causing a lot of attention. It also held crossover marketing activities in China: in April 2015, in Beijing it carried out activities of "Meeting CEOs by one click"; in Shanghai, it carried out "Call a helicopter by one click" activities; in 2015, in the same year it also carried out activities of "Call a sculling boat by one click", "52 Greentown Group executives serving as your Uber drivers", "Get a billion by one click" and " Uber's smart restaurants". Through these crossover marketing activities, Uber gave great experiences to user by tightly grasping their demands and successfully got their attentions, to a greater extent these activities stimulated the users senses of freshness and challenge.

\section{Questionnaire Analysis on Uber's Crossover Marketing}

In July 2017, the author surveyed a lot of Uber's consumers via the network to get the information about their use frequency of Uber apps. The questionnaire was compiled with 15 related questions, and a total of 200 people participated in this survey. The author got 195 effective questionnaires, i.e. an effective rate of $97.5 \%$.

The use frequency of Uber apps. This survey got 195 effective questionnaires, among these people who used Uber apps, there are 125 males and 70 females, accounting for $64.1 \%$ and $35.9 \%$ respectively. A total of $92 \%$ of the surveyed users disclosed that they tried to learn some knowledge about the development history of Uber, it can speculate that the Uber app users have considerable interest on the development history of it. Among the respondents, the users with less than a year of using history since registration accounted for $66 \%$, which shows the considerably fast development speed of Uber in the last year. Among the respondents, most of them disclosed that they mainly use Uber apps as a means of transport, accounting for $97 \%$, which also reflects the considerably fast 
development speed of $\mathrm{O} 2 \mathrm{O}$ taxi in China. Among these respondents, the users with a using frequency of more than 20 times accounted for $81 \%$, which partly reflect that the users of Uber apps have a high reliance on this means.

The survey Results and Analysis of Uber's Crossover Marketing.

Customers' focus on the pattern of Uber's crossover marketing

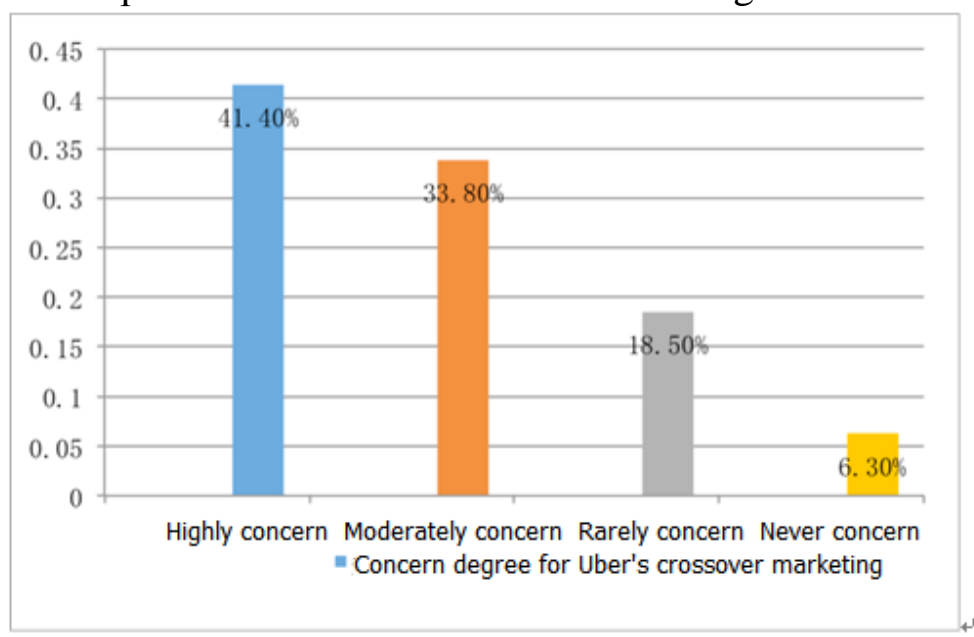

Figure 1. Concern degree for Uber's crossover marketing

These 195 Uber clients showed a total of 94\% concerning rate for Uber's crossover marketing pattern, which partly reflects the superior effect brought by the crossover marketing to Uber. Furthermore, this $94 \%$ concerning rate can also guarantee the reliability of the results of the following sub-surveys about Uber's crossover marketing.

The user-centered degree in Uber's crossover marketing

Table 1 The user-centered degree in Uber's crossover marketing

\begin{tabular}{|c|c|c|c|c|}
\hline Degrees & $\begin{array}{c}\text { Highly } \\
\text { user-centered }\end{array}$ & $\begin{array}{c}\text { Moderately } \\
\text { user-centered }\end{array}$ & $\begin{array}{c}\text { Insufficiently } \\
\text { user-centered }\end{array}$ & $\begin{array}{c}\text { Negatively } \\
\text { user-centered }\end{array}$ \\
\hline Accounting for (\%) & $\mathbf{2 1}$ & $\mathbf{4 6}$ & $\mathbf{2 4}$ & $\mathbf{9}$ \\
\hline
\end{tabular}

Among the people surveyed, only $21 \%$ of clients considered that Uber's crossover marketing is highly user-centered, while crossover marketing is generally based on the user experience, so it is a big problem to make users to feel this.

The necessity of the combination of Uber's crossover marketing and city quality

Table 2 The necessity of the combination of Uber's crossover marketing and city quality

\begin{tabular}{|c|c|c|c|c|}
\hline Necessity grading & Highly necessary & $\begin{array}{c}\text { Moderately } \\
\text { necessary }\end{array}$ & Optional & Unnecessary \\
\hline Accounting for $(\%)$ & $\mathbf{5 6}$ & $\mathbf{2 6}$ & $\mathbf{1 3}$ & $\mathbf{5}$ \\
\hline
\end{tabular}

Among the people surveyed, $82 \%$ of clients considered that the combination of Uber's crossover marketing and city quality is necessary. This reflects that Uber users' positive attitude towards the pursuit of city quality and keeping pace with the times.

The necessity of selecting good cooperation brands in Uber's crossover marketing

Table 3 The necessity of selecting good cooperation brands in Uber's crossover marketing

\begin{tabular}{|c|c|c|c|c|}
\hline Necessity grading & $\begin{array}{c}\text { Highly } \\
\text { necessary }\end{array}$ & $\begin{array}{c}\text { Moderately } \\
\text { necessary }\end{array}$ & Optional & Unnecessary \\
\hline Accounting for $(\%)$ & $\mathbf{5 6}$ & $\mathbf{2 6}$ & $\mathbf{1 3}$ & $\mathbf{5}$ \\
\hline
\end{tabular}


From the survey, users generally believe that Uber should select good cooperation brands in crossover marketing, in order to achieve the greatest publicity benefits. On the purpose of crossover marketing, the marketing campaigns are carried out to cooperate with a number of brands to achieve the effect of win-win outcomes, so Uber is necessary to select good cooperation brands in crossover marketing.

The necessity of breaking with tradition in Uber's crossover marketing

Table 4 The necessity of breaking with tradition in Uber's crossover marketing

\begin{tabular}{|c|c|c|}
\hline Classification & Must break with tradition & Must follow the tradition \\
\hline Accounting for $(\%)$ & $\mathbf{1 0 0}$ & $\mathbf{0}$ \\
\hline
\end{tabular}

Crossover marketing belongs to an innovation on traditional marketing model, but crossover marketing is not simply a two-side or multi-party cooperation and pursues user experience rather than complementation. The users who were surveyed agreed that Uber's crossover marketing should break with the tradition and focus on the user experience. This reflects that the user experience-oriented development pattern of crossover marketing is an irreversible trend.

The survey on the customer demand for the localization of Uber's crossover marketing. Generally speaking, any kind of marketing model from the outside world should be localized in order to get the best effect. In other words, Uber, as a foreign group, should also adapt to local conditions in China. From the survey results, most of the people surveyed think that Uber needs to adapt to local conditions of the Chinese market, which is very consistent with the previous analysis.

\section{The Enlightenment of Uber's Crossover Marketing in the Cities of China}

According to the above investigation and analysis, it can find that these customers who underwent this survey have been focusing on Uber's crossover marketing, reaching $94 \%$, so this study has a good practical significance and the following enlightenment can be gotten:

Crossover marketing must be user-centered. The core point of crossover marketing is to take the users as the center, that is, to always stand on the users' position rather than ignoring the users' feelings. At the same time, in addition to making the users physically satisfied, it also needs to make users psychologically satisfied, in order to let users have more preference on the product or even more rely on it.

For example, in June 10, 2015, in Hangzhou, Uber carried out an activity of "Uber's free delicacy day: taste Uber's free delicacy by one click" and attracted a large number of people to participate. From the user's point of view, this marketing activity really paid great attention to the transport and food needs of users and improved the service from top to bottom and its user-centered concept has been widely recognized.

Crossover marketing must keep abreast of city quality improvement. Crossover marketing should conform to the city quality of a particular city, which not only reflects the localization trend of Uber, but also reflects the local-user-experience-centered essence of crossover marketing. This is can be understood in the practices of real life, that is, if a new thing is going to develop in a city or region, it must meet the needs of its users. For example, raw green onions are usually eaten by users in Hebei or other northern China regions; however, the people in the southern China almost always take green onions as ingredients for cooking. In different cities, according to the local people's customs, styles and habits, Uber's crossover marketing should be realized with the more easily acceptable marketing programs that are in line with the city development trend, the needs of improving people's living standards and enriching people's spiritual life.

Crossover marketing must select good cooperation brands. According to the investigation and analysis, it learned that most of the Uber's customers hope Uber can select good cooperation brands in advance before crossover marketing activities. Of course, these customers want Uber to choose the brands what they really need. The enterprise can choose a good partner which has consistency in resources or user consumption habit or has similarity or common ground in corporate 
culture and corporate philosophy, so as to achieve customer sharing and win-win results.

Crossover marketing must break with the traditional model. In China's traditional crossover marketing model, many activities are lack of new ideas and user experience-centered concept and just simply integrate A and B regardless of their compatibility or even just follow the principle of "BOGOF". There fore, China's traditional crossover marketing model is very backward, Uber should break with this traditional model, and should take the user experience as the center to create success. Uber's crossover marketing breakthrough against traditional model not only needs to include the user-experience-centered concept, but also needs to include the localization process of marketing activities, the pursuit of city quality improvement and the selection of excellent partners.

Crossover marketing must be tailored to local conditions. By insisting on user-centered concept, Uber always carried out appropriate strategic activities in the appropriate places, which is an important reason why Uber can always win in a variety of marketing competitions.

Building an organizational structure adapted to the local form. Unlike other large companies, when Uber entered a new city, it just thinks of its new subsidiary in this city as a start-up and only builds a bi-lingual management team with just 3-10 staffs, and then uses the team to carry out all the marketing activities in the city. This team needs to fully take the local characteristics into account and carry out activities based on local users, so as to promote the development of new subsidiary.

Study on strengthening the crossover marketing environment. Crossover marketing environment needs to be built based on the user experience. In order to improve the user experience; it not only needs to pay attention to the specific nature of each city, but also needs to understand the change of a city in different time, such as the road conditions and other problems. Therefore, no matter from the idea design in initial stage to the promotion and implementation in middle stage, it all needs to make deep and detailed investigations on every place of the city within each time period, so as to get the information needed in the process. Finally, the information should be collected, counted and analyzed to find the target users and cooperative brands, and the formal marketing should be carried out after every factor is nearly reasonable and feasible.

\section{References}

[1] Yan Jingyu. The thinking on Uber's practice of enhancing brand awareness via crossover marketing. Marketing Strategy [J]. 2015.6 (9).

[2] Liu Tao. Uber's "crossover marketing" around the world[N]. Science and Technology Daily, 2015-05-19. (A2)

[3] Yan Hao. Uber's dream: when the future can be called by just one click [EB / OL]. Official News Platform of Center for Social Media Research, School of New Media, Peking University, 2015-4-29.

[4] Li Jiajia. Uber'sfirst one click helicopter calling service in China, Zhao Youting becomes its first flight passenger [EB / OL]. China News, 2015-4-25.

[5] Shao Hui, Zhou Zoran However, do not do hard and wide cast Uber Tong Dawei with a fire [OL]. Jiemian News, 2015-4-8.

[6] Yu Fei. 52 executives of Hangzhou Greentown Group serve as Uber drivers. Maybe the next lucky customer is you. [EB / OL]. Zhejiang online, 2015-5-11.

[7] Tecado. GuoDegang? Uber service allows you to call a comic actor by one click! [N]. Bund Pictorial, 2015-6-25. (B2)

[8] Zhuang Shengchun. "Innovation and Entrepreneurship Education Alliance of China's Universities" was set up, Uber and other technology companies joined it [EB / OL]. CN Radio, 2015-6-12.

[9] Huang Kunming. Adhere to scientific development, enriching the people and improving the city, struggle to build an eastern city full of high quality, happiness and harmony: Hangzhou [R]. The Eleventh Party Congress report of Hangzhou City, 2012.

[10] Wei Junying. Integrated marketing communication: concepts and methods [M]. Zhejiang University Press, 2005: 18-25 
[11] A relay game for 300 cities, Uber makes a lot fun in the world's largest ice cream day [EB / OL]. Huanqiu News, July 22, 2015.

[12] Lin Xi. Uber's valuation reaches 50 billion US dollars and may break a record [EB / OL]. Xinhua News Agency, 2015-05-13. 